\title{
MODELAGEM DO ERRO DEVIDO À ILUMINAÇÃO AMBIENTE NAS MEDIÇÕES OBTIDAS COM CÂMERA DE DISTÂNCIA
}

\section{Modelling of error due to background lighting in measurements obtained from range camera}

\author{
FELIPE MARTINS MARQUES DA SILVA ${ }^{1}$ \\ JORGE ANTONIO SILVA CENTENO ${ }^{1}$ \\ 1Programa de Pós-Graduação em Ciências Geodésicas \\ Universidade Federal do Paraná - Curitiba - Brasil.Email:engfelipemarques21@gmail.com; Email: \\ centeno@ufpr.br
}

\section{RESUMO}

As câmeras de distância são capazes de medir a distância entre o sensor e a superfície dos objetos para cada pixel da imagem, em um único instante, produzindo uma imagem de distâncias. Além das distâncias, também obtém a intensidade e a amplitude para cada pixel, formando as imagens de intensidade e amplitude, respectivamente. Como toda a medida, aquelas obtidas pelas câmeras de distância possuem erros, e um dos fatores que geram erros nas medidas é a iluminação ambiente. Neste estudo foi analisada a influência que a iluminação ambiente exerce sobre os valores calculados de distância, amplitude e intensidade. Para isso, realizou-se experimentos em uma sala escura, onde foram obtidas imagens de distância, intensidade e amplitude de uma parede branca, em que incidia diversos níveis de iluminação gerada por duas lâmpadas cujos feixes convergiam ao centro da parede. Verificou-se que: a iluminação fornecida nos experimentos aumentou os valores de distância; a relação entre os erros de distância e a variação da amplitude é diretamente proporcional e linear; e que a relação entre os erros de distância e a variação da intensidade também se mostrou linear para valores de intensidade menores que 26600, para a frequência de modulação de $21 \mathrm{MHz}$. As correlações lineares atingiram valores acima de 0,9. A imagem de intensidade se mostrou mais sensível à incidência de iluminação ambiente do que a imagem de amplitude.

Palavras-chave: Iluminação ambiente, Câmera de distância, Erros.

\begin{abstract}
:
Range cameras are able to measure the distance between the sensor and the surface of objects for each image pixel, in a just single instant, producing an image of distance. It also measure the intensity and amplitude for each pixel, in order to produce images of intensity and amplitude, respectively. Like any measure, those obtained by the distance of cameras have errors, and one of the factors that produces errors in the measurements is the external lighting at the environment. In this study it was analyzed the influence of the external lighting on the calculated
\end{abstract}


values of distance, amplitude and intensity. In a dark room it was made up some experiments, where it was obtained images of distance images, intensity and amplitude of a white wall, where various lighting levels generated by two lamps whose beams converged on the center of the wall. Some conclusions are: the external illumination increased distance values; the ratio of the distance error and the amplitude variation is directly proportional and linear; and the relationship between the distance error and the variation of intensity it was also linear for values lower intensities than 26600, at $21 \mathrm{MHz}$ of modulation frequency. It was achieved linear correlation above 0.9. The image of intensity is more sensitive to the effect of external lighting than the amplitude image.

Key-words: Background illumination, Range camera, Errors.

\section{INTRODUÇÃO}

As câmeras de distância ou câmaras ToF (Time of Flight) são dispositivos capazes de medir a distância entre o sensor e vários pontos da superfície dos objetos de uma cena, gerando uma imagem de distância, isto é, uma imagem em que para cada pixel há a informação da distância entre a câmera e o alvo. Também pode fornecer a amplitude e a intensidade do sinal refletido pela superfície, em cenas estáticas ou dinâmicas, gerando imagens de amplitude e intensidade, respectivamente (Lichti, 2008).

As imagens de distância, amplitude e intensidade são obtidas simultaneamente, em um único instante, sem a necessidade de utilizar mecanismos de varredura (Lichti, 2008; Foix et al., 2011; Shahbazi et al., 2011). Segundo Lichti (2008), esta é a principal vantagem da câmara de distância sobre os equipamentos de varredura a laser. Outras vantagens que podem ser citadas são: o baixo peso da câmara e o consumo reduzido de energia (Foix et al., 2011); a estrutura compacta com apenas um sensor (Foix et al., 2011; Shahbazi et al., 2011); e a alta taxa de aquisição de imagens, que possibilita o mapeamento e localização em tempo real (Shahbazi et al., 2011). Segundo Lichti (2008) como desvantagens tem-se a baixa resolução e a limitação de distância livre de ambiguidades - a chamada ambiguidade é a quantidade de comprimentos de onda inteiros decorridos entre a emissão do sinal e a sua captação pelo sensor, referindo-se às câmeras de distância baseadas na diferença de fase do sinal. Além destas desvantagens, também pode-se citar a precisão centimétrica das medidas obtidas pela câmera, inferior à obtidas com outras técnicas.

Dentre as possíveis utilizações da câmera, pode-se citar: reconhecimento de gestos ou movimentos humanos; mobilidade de robôs; "reconstrução" de movimentos executados por atletas; modelagem de superfícies; etc.

Assim como todas as observações realizadas com qualquer instrumento, as observações realizadas com as câmeras de distância possuem erros. Tais erros são originados por fatores não ambientais e fatores ambientais. Dentre as fontes de erro que não ambientais - que geram os erros instrumentais - pode-se citar: (1) a temperatura do sensor (Kahlmann et al., 2006; Steiger et al.,2008; Chiabrando et al., 2009; Piatti e Rinaudo, 2012); (2) o Tempo de Integração (Kahlmann et al., 2006; Wiedemann et al., 2008; Foix et al., 2011) e; (3) os materiais de fabricação do sensor (Lange, 2000; Kahlmann et al., 2006; Lindner e Kolb, 2007; Foix et al., 2008).

Já entre as fontes de erro que dependem das características do ambiente, tem-se: (1) a relação sinal-ruído (Liu, 2002; Foix et al., 2011); (2) a amplitude da reflexão dos objetos da cena (Lindner e Kolb, 2007; Falie e Buzuloiu, 2007; Foix et. al., 2011); (3) o multicaminhamento percorrido pelo sinal (Dorrington, 2011; Fuchs, 2012); (4) o espalhamento interno do sinal 
dentro da câmera (Mure-Dubois e Hugli, 2007; Lindner e Kolb, 2007; Kavli, et al, 2008; Karel, 2008; Sabov e Krüger, 2008; May et al., 2009; Jamtshove Lichti, 2010; Reynolds et al., 2011; Lichti, Qi e Ahmed, 2012; Karel et al, 2012; Schafer, Lenzen e Garbe, 2014; Lichti et al, 2014) e; (5) a iluminação ambiente (Möller et al., 2005; Kahlmann, 2007; May et al., 2007; Kazmi, Foix e Aleny`A, 2012; Kazmi et al, 2014).

Além das fontes citadas, ainda pode-se citar o ângulo de incidência do sinal sobre o alvo (Hebert e Krotkov, 1992; Anderson et al., 2005; Kim et al., 2008) e a distância entre a câmera e o alvo (Lindner et al., 2010; Kolb et al. 2010; Foix et al., 2011; Silva e Centeno, 2015).

No presente artigo busca-se modelar os efeitos da iluminação ambiente. Outros pesquisadores também estudaram a incidência da iluminação sobre as observações das câmeras da distância, tais como Möller et al (2005), Kahlmann (2007), May et al (2007), Kazmi, Foix e Aleny`a (2012) e Kazmi et al (2014).

Segundo Möller et al (2005), se a iluminação ambiente for mais intensa que a iluminação ativa da câmera, dois problemas podem ocorrer: (1) ruídos na imagem, ocasionando erros nas medidas das distâncias, uma vez que a relação sinal/ruído (SNR - Signal to Noise Ratio) apresentará menor valor e; (2) saturação do pixel, quando a energia captada é excessiva, impossibilitando o cálculo da distância.

Para aumentar o valor da relação sinal/ruído algumas medidas podem ser tomadas, tais como: (a) ajustar o tempo de integração para evitar a sub-iluminação ou a saturação do pixel; (b) aumentar a quantidade de emissores de energia - LEDs (Light-Emitting Diodes) ou aumentar a sua potência (Kahlmann, 2007); (c) utilizar filtros espectrais (passa banda) para limitar a captação da energia eletromagnética a uma faixa mais estreita do espectro (Möller et al., 2005); (d) utilizar o chamado "modo burst", isto é, aumentar a potência do sinal emitido em um curto espaço de tempo (May et al., 2007).

Por fim, Kazmi, Foix e Aleny`a (2012) e Kazmi et al (2014) realizaram estudos sobre a influência da iluminação oriunda da luz solar, à sombra e em ambiente fechado, sobre folhas de vegetação, buscando encontrar o melhor tempo de integração para cada situação.

Nas pesquisas citadas, os pesquisadores buscaram minimizar os efeitos da iluminação ambiente sobre as medições através do aumento do valor da relação sinal/ruído. Já para a pesquisa mostrada neste artigo, procurou-se verificar a influência da variação da iluminação sobre os valores de distância, amplitude e intensidade calculados pela câmera, procurando inter-relacionar estes valores e modelá-los. Para tal, foi utilizada uma câmera de distância modelo PMD Camcube 3.0.

As próximas seções deste artigo apresentam a fundamentação teórica que embasa a pesquisa (seção 2), os equipamentos utilizados (seção 3), a metodologia proposta (seção 4), a análise dos resultados (seção 5) e as suas conclusões (seção 6).

\section{FUNDAMENTAÇÃO TEÓRICA}

Nesta seção será explicado o funcionamento da câmera de distância PMD, o conceito de tempo de integração e seus efeitos, bem como os efeitos da iluminação ambiente.

\subsection{Funcionamento da câmera de distância}

Bol. Ciênc. Geod., sec. Artigos, Curitiba, v. 22, nํ1, p.54 -72, jan-mar, 2016. 
A câmera de distância opera com um sensor tipo PMD (Photonic Mixer Device), que consiste em um sensor fotoelétrico capaz de armazenar informações sobre a intensidade, a amplitude e a diferença de fase entre o sinal emitido e o sinal recebido.

Este sensor funciona com os mesmos princípios dos sensores CMOS (Complementary MetalOxide-Semiconductor), realizando a conversão de voltagem em carga e amplificação do sinal em cada um dos fotodiodos do sensor. Devido ao fato de cada fotodiodo realizar a conversão/amplificação do sinal, este sensor é mais propenso a ruídos se comparados aos sensores CCD (Charged-Coupled Device). Da mesma foram, apresenta menor consumo de energia e mais rapidez na formação das imagens.

Para cada pixel da imagem, a câmara PMD Camcube 3.0 calcula a diferença de fase entre sinal emitido e o sinal de retorno, permitindo o cálculo da distância. Na Figura 1 são mostradas duas ondas senoidais representando o sinal emitido $g(t)$, em linha cheia, e o sinal recebido ou de retorno $\mathrm{s}(\mathrm{t})$, em linha pontilhada. A amplitude (A) do sinal recebido tende a ser menor que a do sinal emitido, uma vez que sofre o efeito de atenuação ao longo do percurso. Ainda na Figura 1, é mostrada a intensidade (B), a diferença de fase $(\varphi)$ e o comprimento de onda $(\lambda)$.

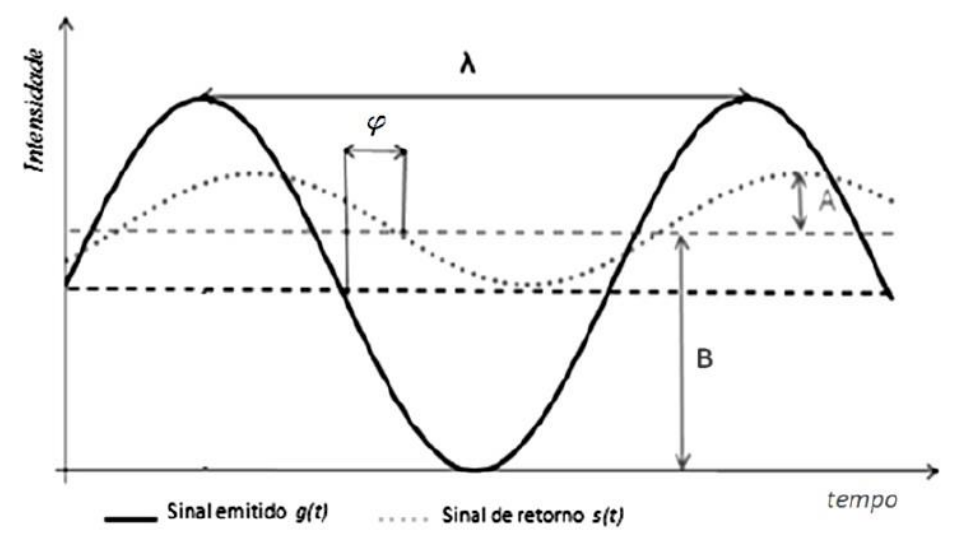

Figura 1: Representação da diferença de fase $(\varphi)$.

Fonte: Adaptado de Piatti (2010).

Sendo $\mathrm{g}(\mathrm{t})$ o sinal modulado emitido pelas fontes de iluminação da câmara, representado matematicamente pela Equação 1, e s(t) o sinal de retorno, representado pela Equação 2, as distâncias em cada pixel são calculadas com base na função de correlação cruzada (FC) entre estes dois sinais (Fuchs e Hirzinger, 2008). Esta correlação cruzada, representada na Equação 3, é utilizada para medir diferença de fase (atraso) entre o sinal emitido e o sinal recebido, permitindo calcular os valores de distância, amplitude e intensidade para cada pixel da imagem (Lange e Seitz, 2001; Lindner e Kolb, 2006; Fuchs e Hirzinger, 2008; Lichti, 2008).

$$
\begin{gathered}
g(t)=\cos (\omega \cdot t) \\
s(t)=k+A \cdot \cos (\omega \cdot t+\varphi) \\
F C(\tau)=s(t) \star g(t)=\lim _{T \rightarrow \infty} \frac{1}{T} \int_{-}^{+T / 2} / 2 s(t) \cdot g(t+\tau) d t=B+\frac{A}{2} \cdot \cos (\omega \cdot \tau+\varphi)
\end{gathered}
$$


sendo: $\mathrm{g}(\mathrm{t})=$ função do sinal emitido; $\mathrm{s}(\mathrm{t})=$ função do sinal recebido (de retorno); $\mathrm{FC}(\tau)=$ função de correlação entre o sinal emitido $\mathrm{g}(\mathrm{t})$ e sinal recebido $\mathrm{s}(\mathrm{t}) ; \tau=$ instantes utilizados para amostras da função de correlação cruzada; $\mathrm{A}=$ amplitude do sinal recebido; $\mathrm{B}=$ intensidade do sinal recebido; $\mathrm{k}=$ constante não modulada, decorrente da iluminação ambiente; $\star$ = operador de correlação cruzada; $\omega=$ frequência angular de modulação do sinal $\mathrm{g}(\mathrm{t})$, onde $\omega=2 \pi f_{\text {mod }} ; f_{\text {mod }}$ é a frequência de modulação do sinal; $\varphi=$ diferença de fase entre o sinal emitido $g(t)$ e $o$ sinal recebido $\mathrm{s}(\mathrm{t}) ; \mathrm{T}=$ tempo de integração; e $\mathrm{t}=$ tempo.

Para efetuar tais cálculos são tomadas quatro amostras da função de correlação cruzada (FC) em instantes distintos $\left(\tau_{0}, \tau_{1}, \tau_{2}\right.$ e $\left.\tau_{3}\right)$, defasados de $\pi / 2$ no tempo, conforme pode ser visto na Figura $2 \mathrm{a}, 2 \mathrm{~b}, 2 \mathrm{c}$ e $2 \mathrm{~d}$. As áreas em azul correspondem à realização da correlação cruzada, por meio da integração da FC. A Figura 2e mostra a curva representativa da função de correlação cruzada.

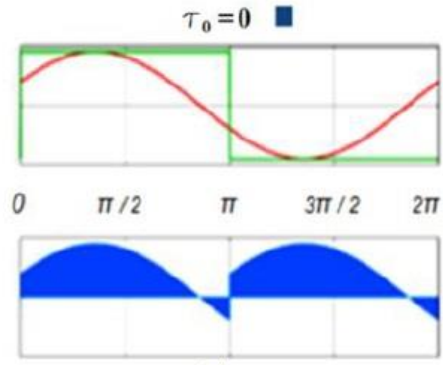

(a)

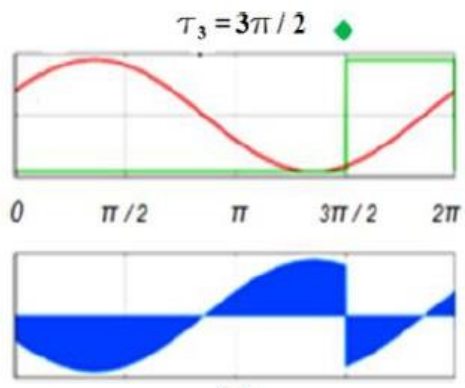

(d)

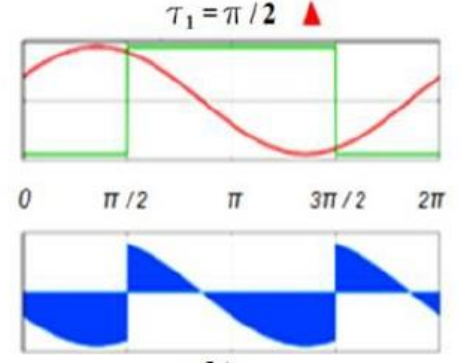

(b)
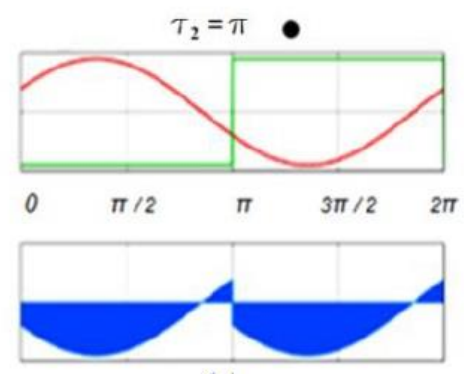

(c)

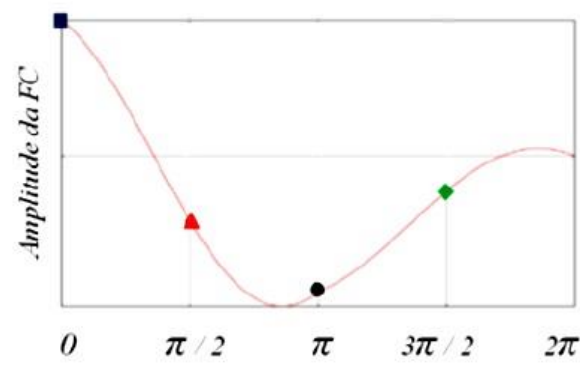

(e)

Figura 2: Representação da correlação cruzada entre o sinal emitido (quadrático) e o sinal de retorno (senoidal) para os instantes (a) $\tau_{0}=0$, (b) $\tau_{1}=\mathrm{p} / 2$, (c) $\tau_{2}=\mathrm{p} \mathrm{e}$ (d) $\tau_{3}=3 \mathrm{p} / 2$; (e) curva representativa da função da correlação cruzada.

Fonte: Adaptado de Rapp (2007)

A partir das equações de FC nos quatro instantes $\tau_{0}, \tau_{1}, \tau_{2}$ e $\tau_{3}$, a diferença de fase é recalculada pela Equação 4 (Lange, 2000; Khalmann et al., 2006).

$$
\varphi=\arctan \left(\frac{F C\left(\tau_{0}\right)-F C\left(\tau_{2}\right)}{F C\left(\tau_{1}\right)-F C\left(\tau_{3}\right)}\right)
$$

Com o valor da diferença de fase pode-se calcular a distância (D) com a Equação 5,

$$
D=\frac{c \cdot \varphi}{4 \cdot \pi \cdot f_{\bmod }}+n \cdot \lambda
$$


onde $c$ é a velocidade da luz no vácuo (299.792.458 m/s); $n$ é o número de ciclos inteiros do sinal (ambiguidade); $f_{\text {mod }}$ é a frequência de modulação do sinal emitido $\mathrm{g}(\mathrm{t})$; e $\varphi$ é a diferença de fase entre o sinal emitido $\mathrm{g}(\mathrm{t})$ e o sinal recebido $\mathrm{s}(\mathrm{t})$.

A primeira parte da equação é a parte fracionada do sinal e é a única utilizada para calcular as distâncias. O termo $n$ - chamado de ambiguidade - representa a quantidade de comprimentos inteiros de onda decorridos entre a câmera e o alvo. Como não é possível calcular seu valor, despreza-se este termo, com o ônus de limitar em apenas um comprimento de onda a distância máxima de medição livre de ambiguidades.

Por exemplo, considerando que o sinal é emitido à velocidade da luz e que a frequência é 20 $\mathrm{MHz}$, o comprimento de onda será de aproximadamente $15 \mathrm{~m}$. Como o sinal tem que percorrer a distância câmara/alvo na ida e na volta, a distância máxima entre o sensor e o alvo fica limitada a $7,5 \mathrm{~m}$, livres da determinação da ambiguidade.

O cálculo da distância, para cada pixel, é realizado através da Equação 6.

$$
D=\frac{c \cdot \varphi}{4 \cdot \pi \cdot f_{\text {mod }}}
$$

O valor de amplitude (A) e de intensidade (B,) para cada pixel, são calculados pelas Equações 7 e 8, respectivamente (Khalmann et al., 2006), sendo que $F C\left(\tau_{0}\right), F C\left(\tau_{1}\right), F C\left(\tau_{2}\right), F C\left(\tau_{3}\right)$ são amostras da $F C$, a cada intervalo de $\pi / 2$.

$$
\begin{gathered}
A=\frac{\sqrt{\left[F C\left(\tau_{0}\right)-F C\left(\tau_{2}\right)\right]^{2}+\left[F C\left(\tau_{1}\right)-F C\left(\tau_{3}\right)\right]^{2}}}{2} \\
B=\frac{F C\left(\tau_{0}\right)+F C\left(\tau_{1}\right)+F C\left(\tau_{2}\right)+F C\left(\tau_{3}\right)}{4}
\end{gathered}
$$

O valor da amplitude não possui uma unidade de grandeza específica, mas seus valores correspondem à quantidade de elétrons captados (Büttgen et al., 2005).

\subsection{Tempo de Integração (TI)}

O Tempo de Integração (TI) consiste no intervalo de tempo disponível para o sensor captar a luz incidente no sensor, sendo similar à velocidade do obturador das câmeras analógicas. No decorrer deste tempo é realizada a integração da função de correlação e quanto maior este tempo, maior será a região abrangida pela integração. 
A mudança do tempo de integração da câmara afeta os valores de distância em todos os pixels da imagem (Foix et al., 2011). A Figura 3 mostra a ocorrência de ruídos nas imagens de distância, intensidade e amplitude, devido à alteração do tempo de integração.

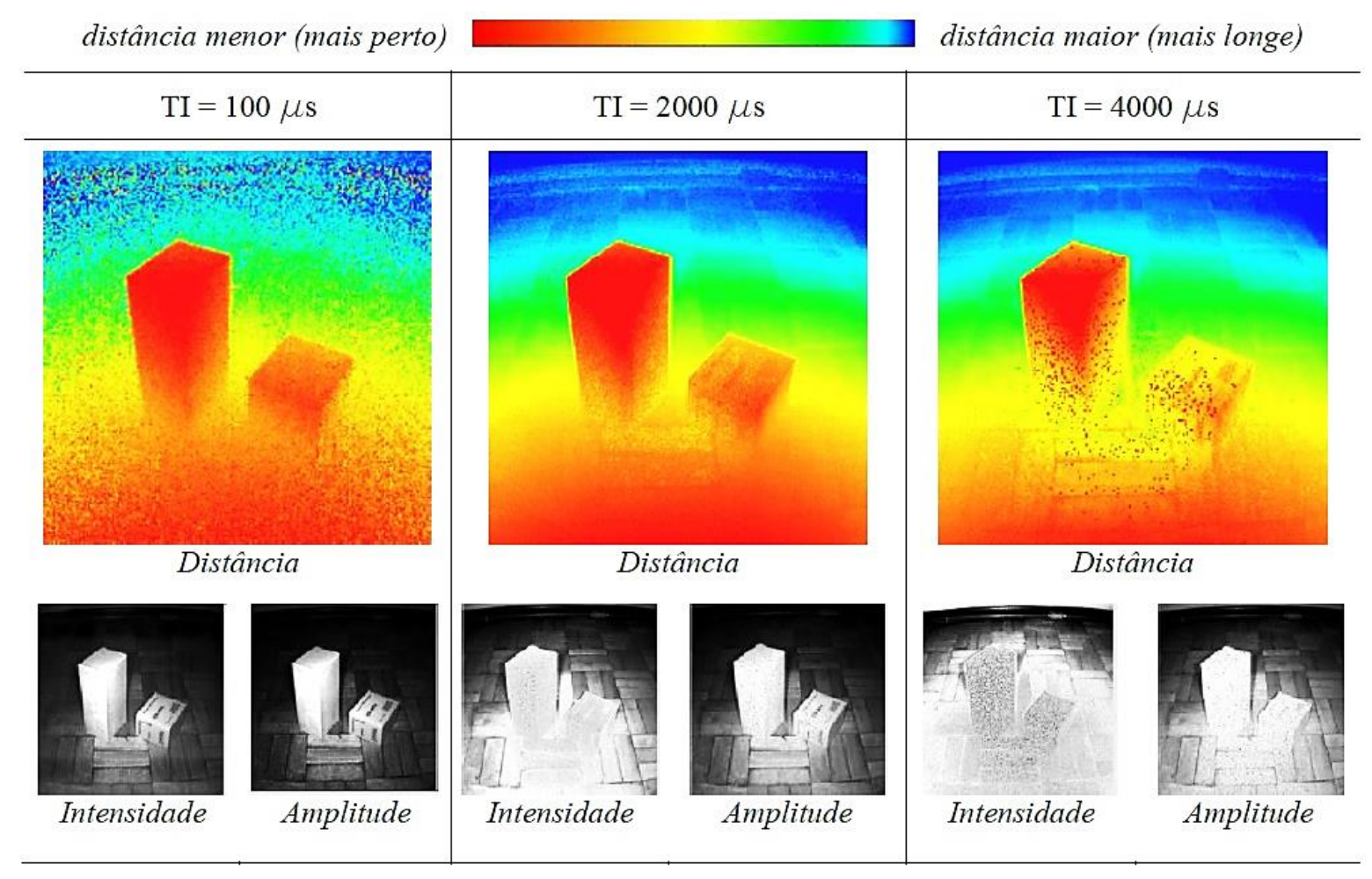

Figura 3: Imagens de distância, intensidade e amplitude para TI 100 ms, 2000 ms e 4000 ms.

Fonte: o autor.

Na Figura 3 a escala hipsométrica indica desde a cor azul, mais distante, até a cor vermelha, mais próximo. Observa-se que para o tempo de integração de 100 microssegundos a imagem de distância apresenta muito ruído, pois o tempo de captação do sinal foi muito baixo. Já para o tempo de integração de 4000 microssegundos ocorre o contrário, com um excesso de captação do sinal, havendo saturação na região central da imagem, gerando ruídos. O tempo de integração de 2000 microssegundos se mostra mais adequado para a cena.

Ambas as imagens, de intensidade e de amplitude, são diretamente proporcionais ao tempo de integração, mas as imagens de intensidade se mostram mais sensíveis a este aumento. Tal verificação é explicada pelas Equações 7 e 8, de amplitude e intensidade, respectivamente, onde a amplitude é calculada se utilizando de diminuições de valores da FC de instantes distintos, havendo uma normalização dos valores. O mesmo não ocorre no cálculo da intensidade.

$\mathrm{Na}$ Equação 9 mostra que o valor da relação sinal/ruído é proporcional a amplitude e inversamente proporcional à intensidade. Uma forma de diminuir o valor da intensidade é diminuindo o valor do TI, o que diminui o efeito da iluminação ambiente e aumenta o valor da SNR.

$$
S N R=\frac{\sqrt{2} \cdot A}{\sqrt{B}}
$$


Entretanto, tal diminuição do valor do TI, também causa uma diminuição no valor da amplitude - diminuindo a SNR - de maneira que há um dilema sobre a simples alteração do valor do TI como forma de melhorar a precisão das medidas. Diante disso Kazmi et al. (2014) afirma que o tempo de integração ideal é aquele que melhor relaciona a amplitude e a intensidade, obtendo a maior exatidão e precisão nas medidas.

\subsection{Iluminação Ambiente}

Assim como a alteração do tempo de integração causa alterações nas imagens de distância, intensidade e amplitude - como visto na Figura 4 - o mesmo ocorre com a alteração da iluminação ambiente presente na cena.

A Figura 4 mostra esta alteração, onde se observam imagens de duas caixas sobre um banco, em três situações: com iluminação no nível 0, (sem iluminação, no escuro); com iluminação no nível 4 (aproximadamente 57\% da iluminação); e com iluminação no nível 7 (100\% de iluminação). As porcentagens referem-se à intensidade luminosa máxima $(2 \times 3680$ candelas $)$ fornecida por duas lâmpadas apontadas em direção aos alvos.

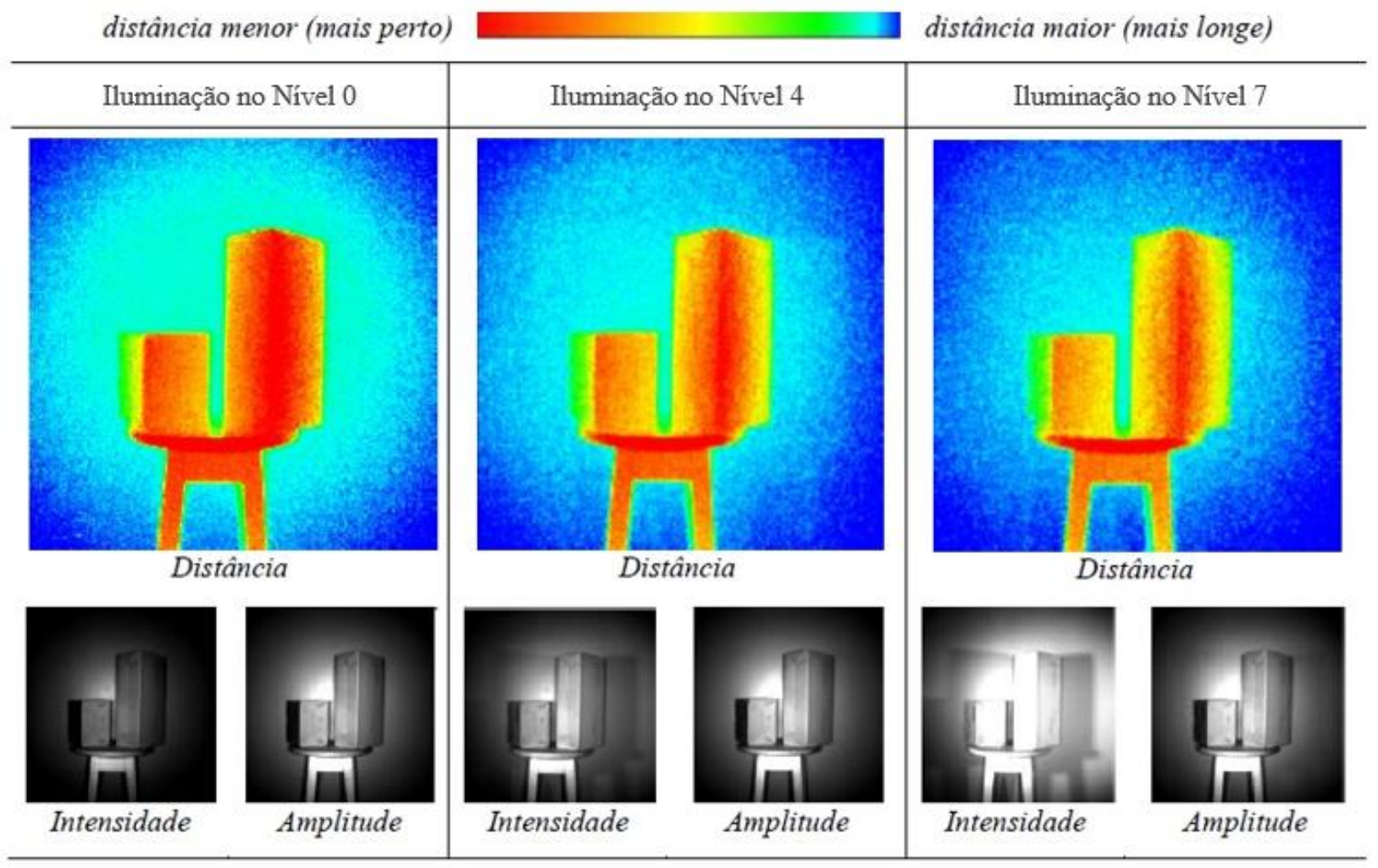

Figura 4: Imagens de distância, intensidade e amplitude para diferentes níveis de iluminação.

Fonte: o autor.

Pelas cores hipsométricas percebe-se que, com a incidência de iluminação ambiente na cena, as distâncias medidas entre a câmera e os objetos se tornaram maiores, sendo observado mais facilmente na parede ao fundo e nas extremidades das caixas. Devido a iluminação, a parede 
fundo apresentou cor azul mais forte, indicando um aumento na distância. Já as extremidades das caixas apresentaram-se menos avermelhadas e mais amareladas, também indicando aumento na distância.

\section{EQUIPAMENTOS}

A seguir são mostrados os equipamentos utilizados neste estudo: a câmara de distância, as lâmpadas halógenas, o dimmer, e o alvo.

\subsection{Câmara PMD CamCube3.0}

Foi utilizada a Câmara PMD CamCube 3.0, mostrada na Figura 5. Observa-se que existem duas fontes de iluminação, uma de cada lado da câmera. Cada fonte de iluminação possui 48 diodos chamados LEDs (Light Emitting Diodes) - que emitem luz no espectro do infravermelho próximo.

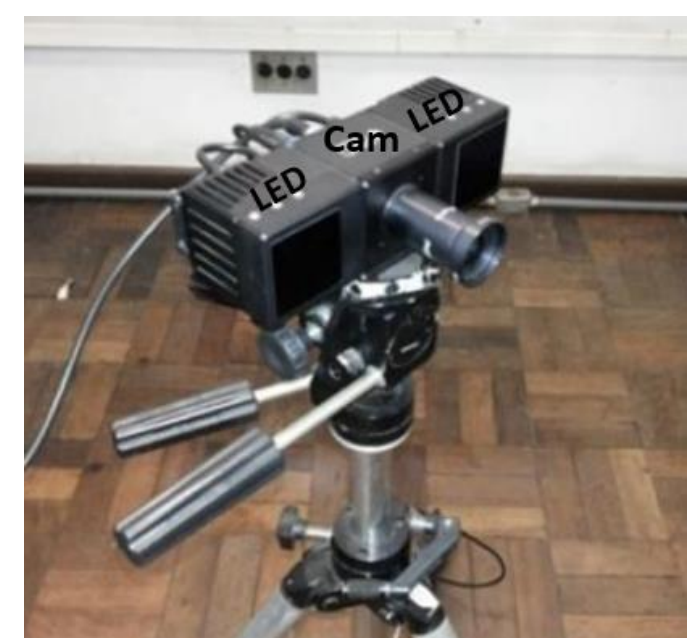

Figura 5: PMD CamCube 3.0 com iluminação LED nas laterais, sobre tripé.

A câmera possui um sensor de imageamento PMD (Photonic Mixer Device), da empresa PMD Technologies, que consiste em um chip que combina as tecnologias CCD e CMOS, com capacidade de armazenar valores de distância de cada pixel. A distância entre o sensor e o alvo é obtida calculando-se a diferença de fase entre o sinal emitido e o sinal recebido. A Tabela 1 apresenta as principais especificações técnicas da PMD Camcube 3.0. 
Tabela 1: Principais especificações técnicas da PMD Camcube 3.0. Fonte: PMD Technologies, 2010.

\begin{tabular}{l|l}
\hline Sensor / Resolução geométrica & $\mathrm{PMD} / 200 \times 200$ pixels \\
\hline Distância focal & $12.8 \mathrm{~mm}$ \\
\hline Campo de Visão & $40^{\circ}(\mathrm{h}) \times 40^{\circ}(\mathrm{v})$ \\
\hline Comprimento de onda da iluminação padrão & $870 \mathrm{~nm}$ \\
\hline Alcance (range) & de $0,3 \mathrm{~m} \mathrm{a} 7,5 \mathrm{~m}$ \\
\hline Taxa de aquisição de imagens (frame rate) & $40 \mathrm{fps}$ \\
\hline
\end{tabular}

Além das características citadas na Tabela 1, a câmara PMD Camcube 3.0 permite escolher as frequências de modulação de $18 \mathrm{MHz}, 19 \mathrm{MHz}, 20 \mathrm{MHz}$ e $21 \mathrm{MHz}$, bem como o tempo de integração da câmara, entre 12 e $50000 \mu \mathrm{s}$. A amplitude do sinal modulado é padrão da câmera. Salienta-se que esta câmera dispõe de uma tecnologia para minimizar os efeitos da luz ambiente nas imagens captadas. É o dispositivo SBI (Suppression of Background Light), cujo objetivo é permitir utilizar a câmera em ambientes externos.

\subsection{Lâmpadas, dimmer e alvo}

Foram utilizadas duas lâmpadas halógenas, modelo Halopar38, marca Osram (Figura 6a), e um dimmer modular universal, marca SkillTec (Figura 6b).

A lâmpada halógena utilizada possibilita a orientação dos feixes de luz com ângulo de abertura do feixe de $30^{\circ}$ - uma vez que possui refletor incorporado (Cavalin e Cervelin, 2007) - fornece intensidade luminosa de 3680 candelas, com potência de $90 \mathrm{~W}$.

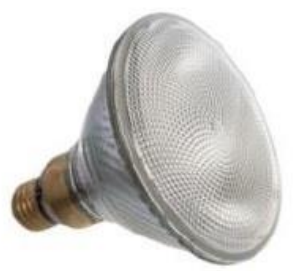

(a)

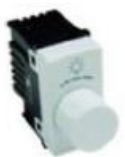

(b)

Figura 6: (a) Lâmpada hálogena Halopar 38 ; (b) Dimmer modular universal.

O dimmer é um tipo de circuito que pode ser montado em um interruptor que, através da diferença de tensão permite regular a corrente que age em um circuito, interferindo no brilho das lâmpadas (Cavalin e Cervelin, 2007). Costuma ser utilizado para controlar a velocidade de ventiladores, a temperatura de chuveiros, etc. Neste estudo o objetivo foi controlar a intensidade luminosa emitida por duas lâmpadas halógenas. O alvo é uma parede de alvenaria, rebocada e pintada na cor branca. 


\section{METODOLOGIA}

Inicialmente foi respeitado o tempo mínimo de 40 minutos para o aquecimento do sensor, necessário para a estabilização das medições realizadas pela câmera, conforme observado em Piatti e Rinaudo (2012) e Silva e Centeno (2015). Quanto ao erro de zero e ao erro de escala, que podem ocorrer em equipamentos de medição eletrônica de distância, verificou-se em Silva e Centeno (2015) que a câmera não é afetada por erro de zero significativo, assim como o erro de escala era desprezível.

A seguir, para a tomada das imagens, a câmera foi posicionada em frente ao alvo, juntamente com as duas lâmpadas posicionadas equidistantes à câmera, uma de cada lado. As lâmpadas foram instaladas na mesma altura da câmera e apontadas de modo a convergir para o centro do alvo (ver Figuras 7a e 7b).

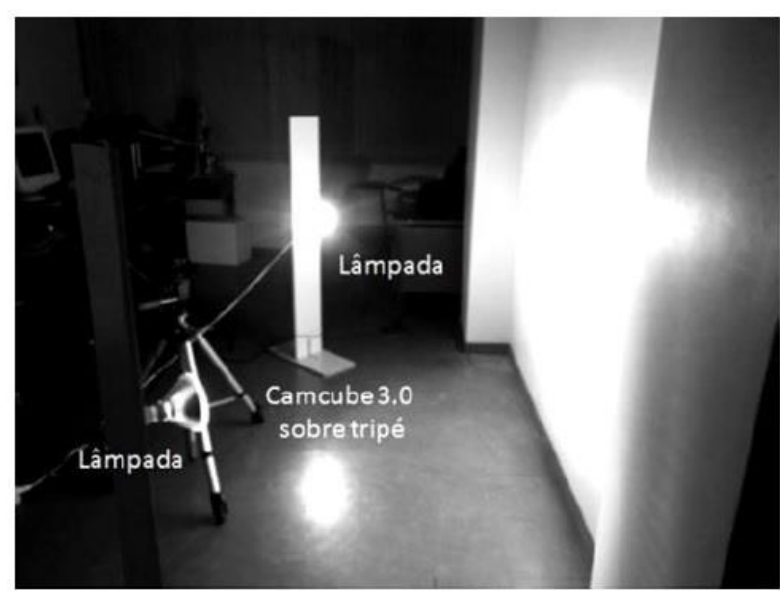

(a)

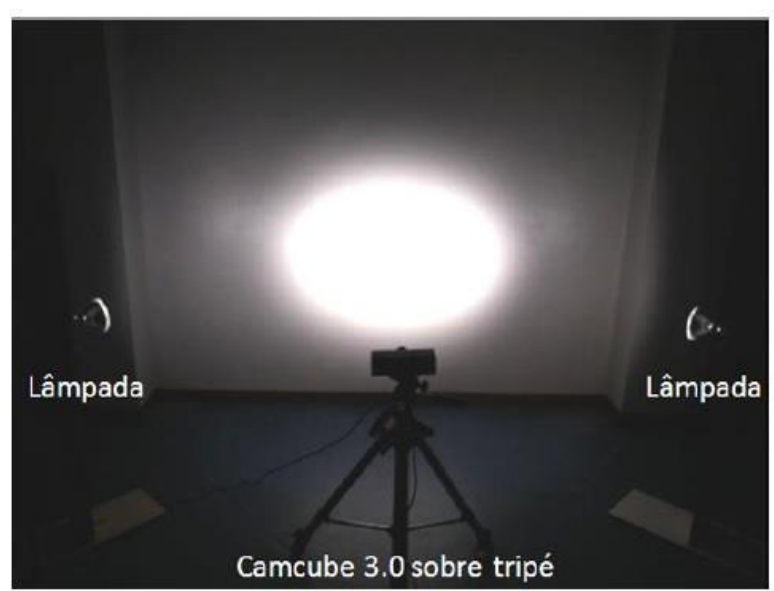

(b)

Figura 7: (a) Visão lateral do experimento; (b) Visão posterior do experimento.

Com esta disposição dos equipamentos, a câmera foi posicionada à $110 \mathrm{~cm}$ do alvo, configurada para emitir sinais com frequências de modulação de 18, 19, 20 e $21 \mathrm{MHz}$, para o TI fixado em 400 microssegundos, uma vez que, para este TI, os erros de distância sem iluminação ambiente (no escuro) foram menores. Alternou-se a intensidade da lâmpada por meio do dimmer, ajustando-se oito níveis de iluminação, desde 0 (escuro) até 7 (iluminação máxima). A tomada das imagens e os resultados foram obtidos utilizando o software Matlab ${ }^{\circledR}$, uma vez que a câmera possui biblioteca própria para este software. Para cada nível foram coletadas 10 imagens (de distância, de intensidade e de amplitude). As configurações para obter as imagens são mostradas na Figura 8.

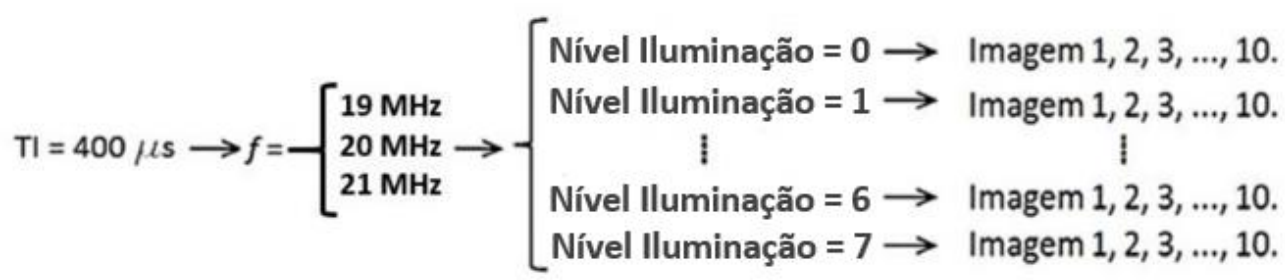

Figura 8: Configuração das coletas das imagens. 
Para cada imagem de distância, intensidade e amplitude calculou-se a média dos pixels de uma janela central de $5 \times 5$, pois a análise de um único pixel central ficaria sujeita aos ruídos que ocorrem na imagem, assim como a análise de uma janela de tamanho maior ficaria sujeita a erros devidos às distorções geométricas causadas pela lente. Para obter os valores dos erros, subtraiuse $110 \mathrm{~cm}$ da distância média calculada, sendo $110 \mathrm{~cm}$ a distância assumida como verdadeira.

Analisou-se a relação entre a variação dos erros de distância e a variação da intensidade, bem como entre a variação dos erros de distância e a variação da amplitude.

\section{ANÁLISE DE RESULTADOS}

Inicialmente, visando mostrar o afastamento da imagem causado pela incidência da iluminação, tem-se a Figura 10, onde há a representação da linha central de duas imagens, com frequência de modulação de $20 \mathrm{MHz}$ : a linha cheia, em vermelho, é a linha central da imagem no escuro; a linha pontilhada, em verde, é a linha central da imagem iluminada a 100\%. O alvo é uma parede branca.

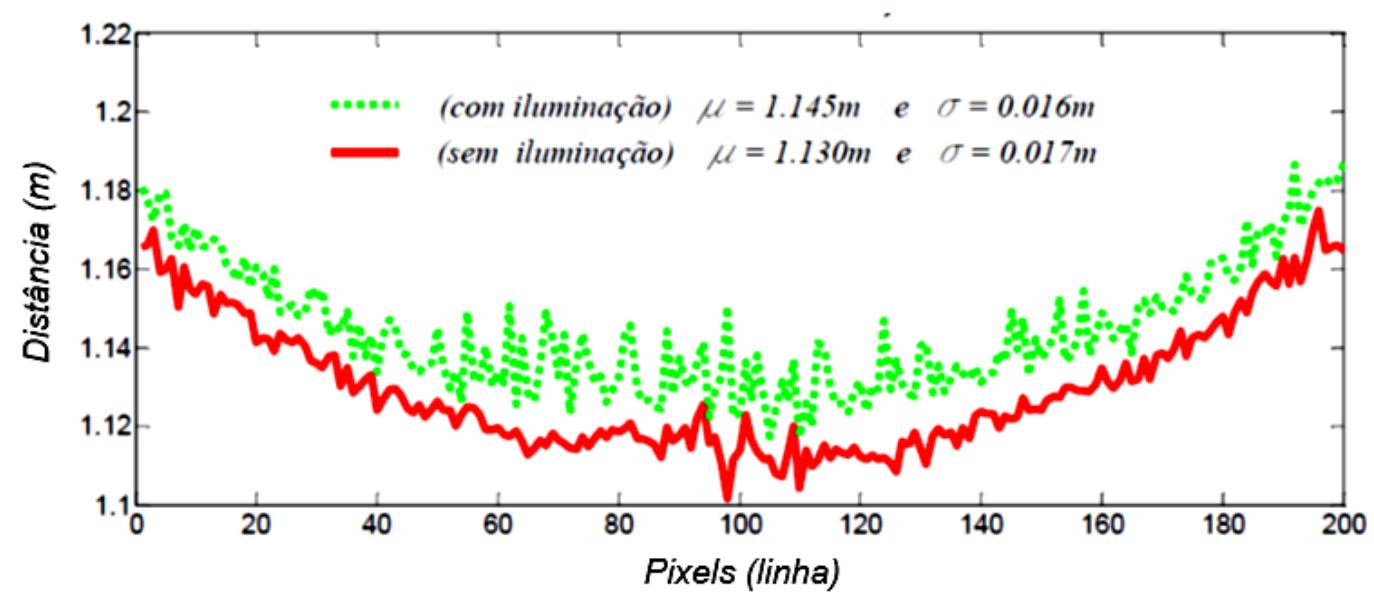

Figura 10: Representação da linha central de duas imagens de distância. Observa-se o afastamento devido à iluminação ambiente.

Observou-se um afastamento da imagem, cuja distância média aumentou de 1,130m para $1,145 \mathrm{~m}$. O desvio padrão calculado apresenta pouca alteração - de $0,017 \mathrm{~m}$ para $0,016 \mathrm{~m}$ - pois a iluminação era focada no centro da imagem e o cálculo foi feito para a linha inteira, ao longo de toda a imagem. É possível observar que a linha com iluminação apresenta maior variação nos valores de distância, representando o ruído na imagem devido à iluminação ambiente. Diante disso verifica que, apesar da câmera PMD Camcube 3.0 possuir o dispositivo SBI, o mesmo não impediu que iluminação ambiente interferisse nos valores registrados nas imagens.

A Figura 11a mostra a relação entre o erro de distância e a intensidade para a frequência de modulação de $19 \mathrm{MHz}$. Não foi possível ajustar os dados a nenhuma equação, uma vez que os valores de intensidade registrados para diferentes níveis de iluminação se repetiram, de modo que, para um mesmo valor de intensidade registra-se dois valores diferentes de erro. Tais conjuntos de dados são representados por elipses pontilhadas na Figura 11a. 


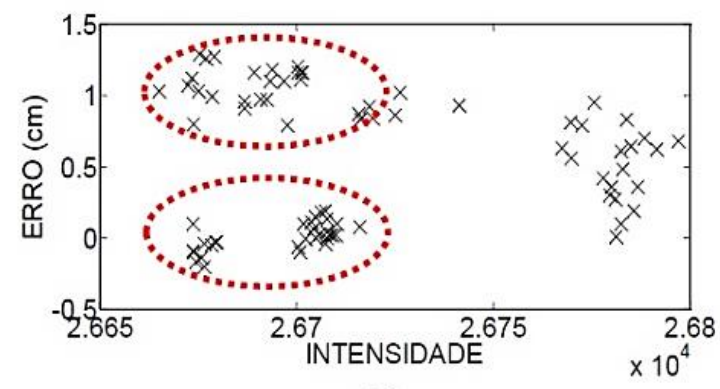

(a)

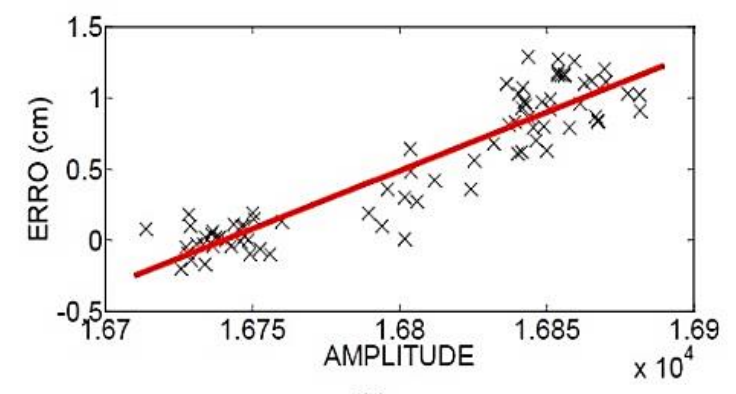

(b)

Figura 11: Para $f_{\text {mod }}=19 \mathrm{MHz}$ : (a) Intensidade x Erro ; (b) Amplitude x Erro.

A Figura 11b mostra a relação entre o erro e a amplitude para a frequência de modulação de $19 \mathrm{MHz}$, que foi modelada através de uma equação de $1^{\circ}$ grau (Equação 10 ), com coeficiente de correlação linear (r) de 0,928 , indicando forte correlação linear positiva.

$$
\operatorname{Erro}(A)=0,00818858 \cdot A-137,08085943 \quad(r=0,928)
$$

Utilizou-se de 8 algarismos decimais devido às diferenças de magnitudes das medidas de erro e de intensidade ou amplitude. Optou-se por manter os valores originais registrados nas imagens, sem qualquer tipo de normalização.

A repetição de valores de intensidade deve-se à saturação causada pela iluminação ambiente excessiva. Através das Figuras 12a e 12b, observa-se que há um afundamento na região central da imagem de intensidade.

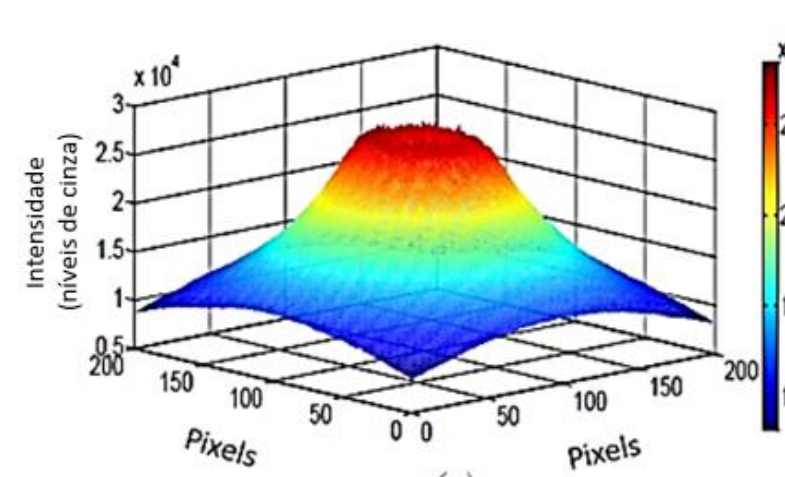

(a)
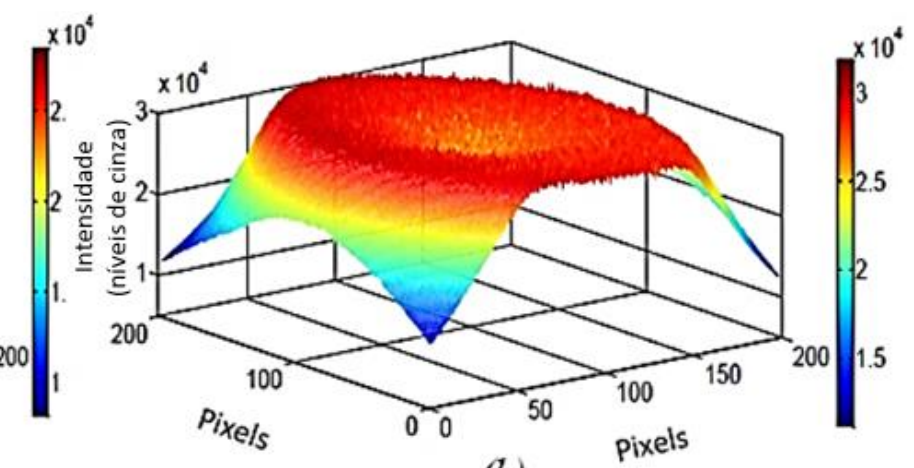

(b)

Figura 12: Representação de duas imagens de intensidade: (a) imagem tomada no escuro; (b) imagem tomada sob iluminação máxima: aumento nos valores de intensidade e saturação na região central.

A saturação ocorreu para valores de intensidade acima de 26600, sugerindo que as imagens de intensidade da câmera PMD Camcube 3.0 são obtidas com resolução radiométrica máxima próxima a 15 bits (para $\log _{2} x=26600$, tem-se $x \cong 14,7$ ), indo de encontro com Kazmi et al (2014), que afirma que imagens de intensidade possuem resolução radiométrica entre 8 e 16 bits. Já na Figura 13, observa-se que não houve saturação na imagem de amplitude. No centro da imagem, onde o sinal emitido é mais forte, a amplitude é maior (em vermelho), sem o afundamento ocorrido na imagem de intensidade mostrada na Figura 12b. Em direção aos bordos 
da imagem, radialmente, a amplitude diminui conforme diminui a incidência do sinal emitido. Imagens de amplitude não possuem unidade definida, sendo proporcional à quantidade de fótons captados em cada pixel (Kazmi et al, 2014).

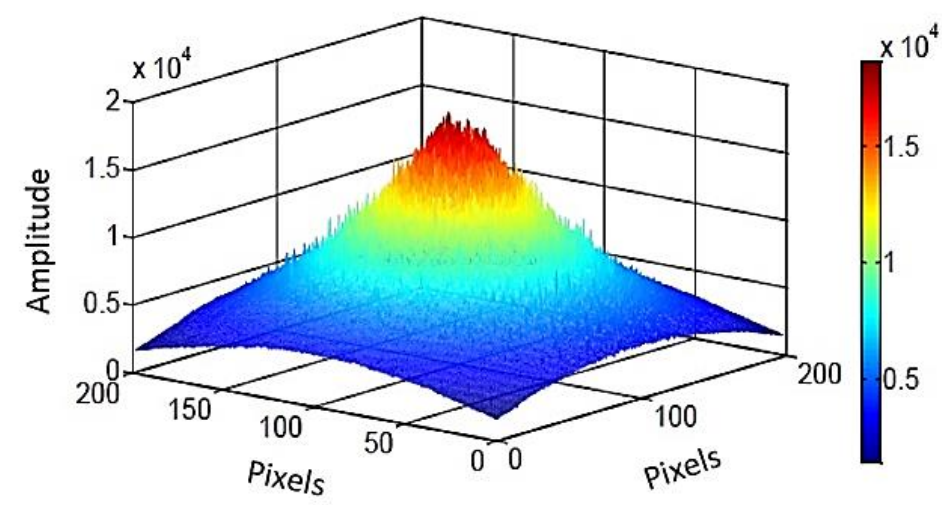

Figura 13: Representação da imagem de amplitude, sob iluminação máxima.

A Figura 14a mostra a relação entre o erro e a intensidade para a frequência de modulação de 20 MHz. Assim como para $19 \mathrm{MHz}$, também não foi possível ajustar os dados a nenhuma equação pois, para um mesmo valor de intensidade, registra-se dois valores diferentes de erro. Tais conjuntos de dados são representados por elipses pontilhadas na Figura 14a. Novamente, a repetição dos valores de intensidade é justificada pela saturação devido à iluminação ambiente excessiva, ilustrada na Figura $12 b$.

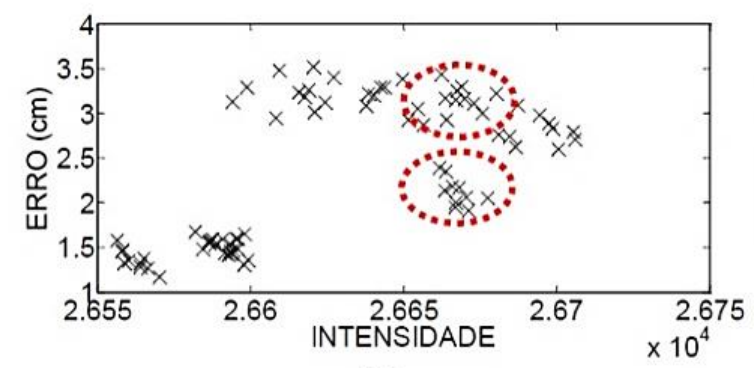

(a)

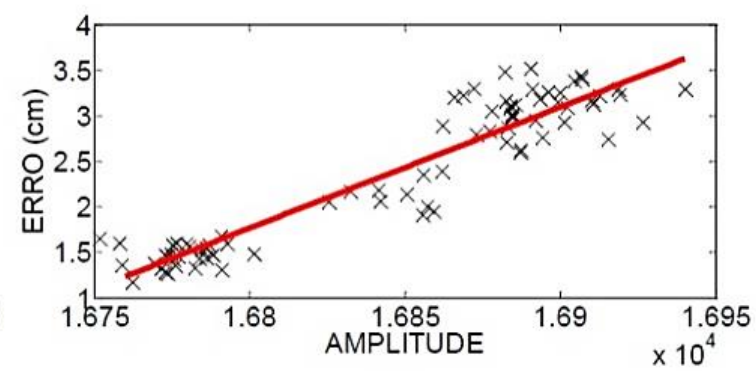

(b)

Figura 14: Para $f_{\text {mod }}=20 \mathrm{MHz}$ : (a) Intensidade $\mathrm{x}$ Erro ; (b) Amplitude $\mathrm{x}$ Erro.

Já na Figura 14b mostra-se a relação entre o erro e a amplitude para a frequência de modulação de $20 \mathrm{MHz}$, que foi modelada através de uma equação de $1^{\circ}$ grau (Equação 11), com coeficiente de correlação linear de 0,941 , indicando forte correlação linear positiva.

$$
\operatorname{Erro}(A)=0,01326741 \cdot A-221,12478026 \quad(r=0,941)
$$

Pelas Figuras 15a e 15b verifica-se que, para a frequência de modulação de $21 \mathrm{MHz}$, o erro em função da intensidade ou em função da amplitude, foi modelado através equações de $1^{\circ}$ grau Equações 12 e 13, respectivamente -, obtendo valores altos para o coeficiente de correlação, uma vez que a dispersão dos valores dos erros é menor. 


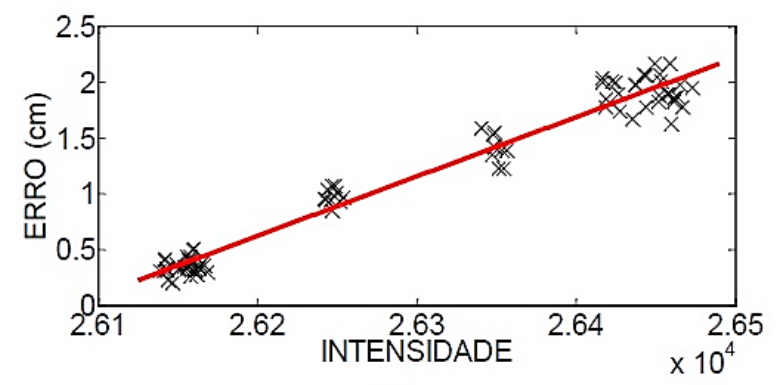

(a)

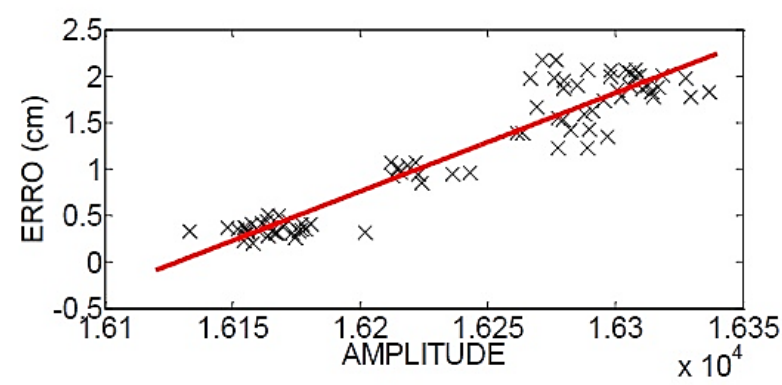

(b)

Figura 15: Para $f_{m o d}=21 \mathrm{MHz}$ : (a) Intensidade x Erro; (b) Amplitude x Erro.

Assim, para a frequência de modulação de $21 \mathrm{MHz}$ foi possível modelar os erros através da variação da intensidade ou da variação da amplitude. Tanto a amplitude, quanto a intensidade, apresentaram forte correlação linear positiva com o erro de distância - 0,951 e 0,983, respectivamente.

$$
\begin{array}{ll}
\operatorname{Erro}(A)=0,01059152 \cdot A-170,82353903 & (r=0,951) \\
\operatorname{Erro}(B)=0,00532633 \cdot B-138,92632435 & (r=0,983)
\end{array}
$$

Os modelos apresentados foram obtidos em ambiente controlado, com as configurações descritas. Para diferentes configurações, os coeficientes da reta poderão se alterar, necessitando calibração específica.

\section{CONCLUSÕES}

A incidência da iluminação influenciou os valores de distância calculados pela câmera, de modo que, quanto maior a intensidade da iluminação, maior o valor de distância calculada. Em outras palavras, a iluminação ambiente aumentou os valores de distância.

Verificou-se que os erros de distância e a variação da amplitude é apresentaram forte correlação linear positiva, com valores acima de 0,9 . A relação entre os erros de distância e a variação da intensidade também apresentou forte correlação linear positiva para valores de intensidade menores que 26600 - também com valores acima de 0,9 - como ocorreu para a frequência de modulação de $21 \mathrm{MHz}$. Para valores de intensidade acima de 26600, houve a saturação da imagem de intensidade, sugerindo que as imagens de intensidade da câmera PMD Camcube 3.0 são obtidas com resolução radiométrica máxima próximo a 15 bits.

A imagem de intensidade foi mais sensível à incidência de iluminação ambiente do que a imagem de amplitude. Assim, a imagem de amplitude se mostrou mais apropriada para a correção dos erros de distância, pois para as três frequências testadas não houve saturação da imagem. 
Os modelos foram obtidos em ambiente controlado. Para diferentes configurações, os coeficientes da reta poderão se alterar, necessitando calibração específica. As características das fontes de erro que incidem sobre as medidas obtidas com as câmeras de distância, principalmente devido à distância câmera-alvo e ao tempo de integração utilizado, exigem a fixação de algumas variáveis.

Para experimentos futuros recomenda-se a verificação dos erros para toda a imagem e a modelagem para iluminação solar, em ambiente externo.

\section{AGRADECIMENTO}

Agradecemos ao CNPq - Conselho Nacional de Desenvolvimento Científico e Tecnológico pela concessão de bolsa de estudos, que permitiu a realização deste trabalho.

\section{BIBLIOGRAFIA}

Büttgen, B.; Oggier, T.; Lehmann, M.; Kaufmann, R.; Lustenberger, F. CCD/CMOS Lock-In Pixel for Range Imaging: challenges, limitations and state-of-the-art. In: $1^{\text {st }}$ Range Imaging Research Day, Zurich, Switzerland, 2005, pp. 21-32.

Cavalin, G.; Cervelin, S. Instalações Elétricas Prediais - conforme a Norma NBR 5410:2004. Ed. Érica, $17^{\mathrm{a}}$ ed., São Paulo, 2007.

Chiabrando, F.; Chiabrando, R.; Piatti, D.; Rinaudo, F. Sensors for 3D imaging: metric evaluation and calibration of a CCD/CMOS time-of-flight camera. Sensors, v.9, pp. 1008010096, 2009.

Dorrington, A. A.; Godbaz, J. P; Cree, M. J.; Payne, A. D.; Streeter, L. V. Separating true range measurements from multi-path and scattering interference in commercial range cameras. Proc. SPIE-IS\&T Electronic Imaging, 2011, v. 7864, 786404-1.

Falie, D.; Buzuloiu, V. Noise Characteristics of 3D Time-of-Flight Cameras. IEEE International Symposium on Signals, Circuits and Systems, 13-14 July, Romenia, 2007, pp. 1-4.

Foix, S.; Alenya, G.; Torras, C. Lock-in Time - of - Flight (ToF) cameras: a survey. IEEE Sensors Journal, v. 11, n. 3, pp. 1-11, 2011.

Fuchs, S.; Hirzinger, G. Extrinsic and depth calibration of TOF-cameras. In IEEE Conference on Computer Vision and Pattern Recognition (CVPR), Anchorage, USA, 2008.

Fuchs, S. Calibration and multipath mitigation for increased accuracy of time-of-flight camera measurements in robotic applications.174 p. Tese (Doutorado em Engenharia). Faculdade de Engenharia Elétrica e Ciência da Computação, Universidade Tecnológica de Berlim, 2012.

Hebert, M.; Krotkov, E. 3D measurements from imaging laser radars: How good are they? International Journal of Image and Vision Computing, Vol. 10, No. 3, pp. 170-178, April, 1992. 
Jamtsho, S.; Lichti, D. Modelling scattering distortion in 3D range camera. International Archives of Photogrammetry, Remote Sensing and Spatial Information Sciences, v.38, pp.299- 304, 2010.

Kazmi, W.; Foix, S.; Aleny`A, G. Plant leaf imaging using time of flight camera under sunlight, shadow and room conditions. Proceedings of 2012 IEEE International Symposium on Robotic and Sensors Environments. p. 192-197, Magdeburg, Alemanha, 2012.

Kazmi, W.; Foix, S.; Alenya, G., Andersen, H. J. Indoor and outdoor depth imaging of leaves with time of flight and stereo vision sensors: analysis and comparison. ISPRS Journal of Photogrammetry and Remote Sensing, v.88, pp.128-146, 2014.

Karel, W. Integrated range camera calibration using image sequences from hand-held operations. The international archives of photogrammetry, remote sensing and spatial Information Sciences, V. XXXVIII, Part 5, Beijing, 2008.

Karel, W.; Ghuffar, S. ; Pfeifer, N. Modelling and compensating internal light scattering in time of flight range cameras. The photogrammetric record, n. 27, v. 138, pp. 155-174, 2012.

Kavli, T; Kirkhus, T; Thielemann, J. T.; Jagielski, B. Modelling and compensating measurement errors caused by scattering in time-of-flight cameras. In Two-and-Three-Dimensional Methods for Inspection and Metrology VI, v. 706604, pp. 1-10, 2008.

Khalmann, T. Range imaging metrology: investigation, calibration and development. Tese de Doutorado em Ciências, Universidade de Hannover, Germany, 2007.

Khalmann, T.; Ingensand, H.; Remondino, F. Calibration for increased accuracy of the range imaging camera swissranger. ISPRS Archives - Volume XXXVI, Part 5, ISPRS Commission V Symposium Image Engineering and Vision Metrology, pp.25-27, Germany, September, 2006.

Kim, Y. M; Chan, D.; Theobalt, C.; Thrun, S. Design and Calibration of a Multi-view TOF sensor fusion system. Computer Vision and Pattern Recognition Workshops, IEEE Computer Society Conference, 2008, pp.1-7.

Kolb, A.; Barth, E.; Koch, R., Larsen, R. Time-of-Flight Cameras in Computer Graphics. Computer Graphics Forum, v. 29, pp. 141-149, 2010.

Lange, R. 3D Time-of-Flight Distance measurement with custom solid-state image sensors in CMOS/CCD-Technology. 139p. Dissertation (Doctor of Technical Sciences). Department of electrical engineering and computer science at University of Siegen, Germany, 2000.

Lange, R.; Seitz, P. Solid state time-of-flight range camera. IEEE Journal of quantum electronics, v. 37, n. 3, 2001.

Lichti, D. Self-calibration of a 3D range camera. The International Archives of the Photogrammetry, Remote Sensing and Spatial Information Sciences, v. XXXVII, Part B5, Beijing, 2008.

Lichti, D., Chow, J. C. K.; Mitishita, E.; Centeno, J.A.S.; Silva, F. M. M.; Barrios, R. A.; Contreras, I. New models for scattering bias compensation in time-of-flight range camera self calibration. Jornal of Surveying Enginnering. American Society of Civil Engineers (ASCE), 2013. 
Lichti, D.; Qi, X.; Ahmed, T. Range camera self-calibration with scattering compensation. ISPRS Journal of Photogrammetry and Remote Sensing v. 74, pp. 101-109, 2012.

Lindner, M.; Kolb, A. - Calibration of the intensity-related distance error of the PMD TOFCamera. In: SPIE - Intelligent Robots and Computer Vision XXV, pp. 6764-35, 2007.

Lindner, M.; Kolb, A. - Lateral and Depth Calibration of PMD - Distance Sensors. In ISVC, Ed. Springer, v.2, pp. 524-533, 2006.

Lindner, M.; Schiller, I.; Kolb, A.; Koch, R. Time-of-flight sensor calibration for accurate range sensing. Journal Computer Vision and Image Understanding, v. 114, issue 12, pp. 13181328, Elsevier Science Inc. New York, USA, Dezembro, 2010.

Liu, X. Cmos image sensors dynamic range and SNR enhancement via statistical signal processing. 139p. Tese (Doutorado em Filosofia) - Department of electrical engineering and the committee on graduate studies of Stanford University, USA, 2002.

May, S.; Pervoelz, K.; Surmann, H. 3D Cameras: 3D Computer Vision of wide scope. Vision Systems: Applications, Edited by: GoroObinata and Ashish Dutta, pp.181-202, Austria, 2007.

May, S.; Fuchs, S.; Droeschel, D.; Holz, D.; Nüchter, A. Robust 3D - Mapping with time-offlight cameras. International Conference on Intelligent Robots and Systems, USA, 2009, pp.1115 .

Möller, T.; Kraft, H.; Frey, J.; Albrecht, M.; Lange, R. Robust 3D measurement with PMD sensors. In: Proceedings of the $1^{\text {st }}$ RangeImaging Research Day, ETH Zurich, Switzerland, 2005.

Mure-Dubois, J.; Hugli, H. Real-time scattering compensation for time-of-flight camera. In Proceedings of the ICVS Workshop on Camera Calibration Methods for Computer Vision Systems - CCMVS2007, 2007, pp. 117-122.

Piatti, D. Time-of-Flight cameras: tests, calibration and multi-frame registration for automatic 3D object reconstruction. 160p. Tese.Doctoral school of Environment and Territory, Politecnico di Torino, Torino, 2010.

Piatti, D.; Rinaudo, F. SR-4000 and CamCube3.0 Time of Flight Cameras: Tests and Comparison. Remote Sensing, v.4, pp.1069-89, 2012.

PMD Technologies. PMD vision CamCube 3.0 Datasheet, v. no. 20100601, 2010.

Rapp, H. Experimental and Theoretical Investigation of Correlating TOF-Camera Systems. Thesis in Physics. Düsseldorf, Germany, 2007.

Reynolds, M.; Dobos, J.; Peely, L.; Weyrich, T.; Brostow, G. J. Capturing Time-of-Flight Data with Confidence. IEEE Conference on Computer Vision and Pattern Recognition (CVPR), 2011, pp. 945-952, Junho, 2011.

Sabov, A.; Krüger, J. Identification and correction of flying pixels in range camera data. Spring Conference on Computer Graphics (SCCG), Slovakia, 2008, pp.135-142.

Schafer, H; Lenzen, F.; Garbe, C. S. Model based scattering correction in time-of-flight cameras. Optics Express, v. 22, issue 24, pp. 29835-29846, 2014. 
Shahbazi, M.; Homayouni, S.; Saadatseresht, M.; Sattari, M. Range camera self-calibration based on integrated bundle adjustment via joint setup with a 2D digital camera. Sensors. v.11, pp. 8721-8740, 2011.

Silva, F. M. M.; Centeno, J. A. S. Modelagem do erro sistemático de distância nas medições realizadas com a câmera de distância PMD Camcube 3.0. Boletim de Ciências Geodésicas. v.21, n.1, pp.141-163, Jan-Mar, 2015.

Steiger, O; Felder, J.; Weiss, S. Calibration of time-of-flight range imaging cameras. Image Processing, ICIP 2008. 15 ${ }^{\text {th }}$ IEEE International Conference on San Diego, CA, 2008.

Wiedemann, M.; Sauer, M.; Driewer, F.; Schilling, K. Analysis and characterization of the PMD camera for application in mobile robotics. Proceedings of the 17th World congress the international federation of automatic control, Korea, July 6-11, 2008.

Recebido em março de 2015.

Aceito em setembro de 2015. 